\title{
Introducing Cell-Plasticity Mechanisms into a Class of Cell Population Dynamical Systems
}

\author{
Walid Djema \\ Catherine Bonnet \\ Frédéric Mazenc \\ Jean Clairambault
}

\begin{abstract}
Cells have amazing features that allow them to guide their development paths and determine their individual and collective fates. Dedifferentiation and transdifferentiation (cell plasticity) are littleunderstood phenomena that allow cells to regress from an advanced differentiated state to a less differentiated one, including the case where cells lose their specific function and become stem cells. In this paper, we introduce cell plasticity into a class of mathematical models we are interested in. We explore a new model involving a dedifferentiation function in the case of two cell maturity stages (stem cells and progeny). We highlight the role that dedifferentiation may have in the survival of cancer cells during therapy. The latter hypothesis appears to be in line with some medical observations.
\end{abstract}

Key Words: Delay, Nonlinear, Cellular plasticity.

\section{INTRODUCTION}

1) Stem cells (SCs): SCs are undifferentiated cells characterized by their ability to self-renew and their multipotency, which is the ability to differentiate into more specialized cells. A SC that engages in the division process (the cell cycle) undergoes a series of transformations until becoming two daughter cells. Most of the cells are in a non-proliferative phase, known as $G_{0}$ or resting phase. A resting cell prepares its entrance into the cell cycle, which consists of four consecutive discrete subphases $G_{1}, S, G_{2}$ and $M$. The M phase is mitosis (nuclear division) that ends with cytokinesis, which is the final step of production of two daughter cells ([7], Chap. 17). In view of the broad range of options available for SCs, including: self-renewal, differentiation, lineage specification, programmed cell death (apoptosis), and quiescence $G_{0}$, determining the fate of a given SC becomes a key challenge [14]. In particular, it is agreed that a better understanding of the processes guiding the fates of SCs may elucidate the causes of cancer and the rise of Cancer Stem Cells (CSCs) (see, e.g., [24], [14]).

The mathematical modeling and analysis become crucial in the query of understanding cancer [22]. In general, the ultimate goal behind theoretical studies of biological systems is to firmly grasp their healthy or unhealthy

Walid Djema* corresponding author, walid.djema@inria.fr. Walid Djema, Catherine Bonnet and Frédéric Mazenc are with Inria, University Paris-Saclay, L2S (CNRS), CentraleSupélec, Gifsur-Yvette, France. Jean Clairambault is with Inria, University of Sorbonne, UPMC, Laboratory Jacques-Louis Lions, Paris, France. walid.djema@inria.fr, frederic.mazenc@inria.fr catherine.bonnet@inria.fr, jean.clairambault@inria.fr behaviors [21], including mechanisms governing cell population dynamics (as for blood cell dynamics [2], [10]). The modeling and analysis approaches are in fact further complicated by some new biological evidences, that enhance cell features and expand their fate possibilities, such as dedifferentiation $^{1}$ (see [32], [6], [15] [18], [17]).

2) Cell plasticity: It has been believed that once a cell differentiates into a particular cell type that has a distinctive function (e.g. when an hematopoietic SC differentiates into one type of white blood cells), it permanently loses the potential for diverse functions and stably maintains its identity [32]. The discovery of dedifferentiation contradicted this biological postulate, and thus opening the way to a much richer and complex cellular behavior. In addition, it was also proved that adult SCs may first reside in one place and then contribute to another tissue [29]. This phenomenon is known as transdifferentiation ${ }^{2}$. In fact, when the process of cell generation and continuous replenishment is perturbed (e.g. tissue injury or hemorrhage), the homeostatic mechanisms are invoked to allow adequate regeneration of damaged tissues [29]. Cell plasticity is already used to produce SCs in regenerative medicine ${ }^{3}$ [14], thus avoiding the ethical issues that usually arise regarding the use of embryonic SCs in research [6]. Cell plasticity provides also new hypotheses for the origin of cancer and some insights into its treatment (see, e.g., [13], [15], [18], [17]).

3) Objectives and organization of the work: We aim to deepen the analysis of cell dynamics, by taking into account the process of cell dedifferentiation in living organisms, in the class of systems as in [20], [1]. More precisely, we consider a general model, that includes a resting and proliferating phases as in [20], [5], and which takes into account several discrete cellmaturity stages, as in [1]. Then, we extend the model through the addition of some cell plasticity mechanisms. In fact, since this is the first time cellular plasticity is

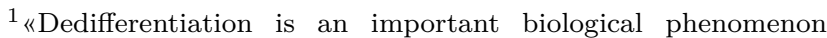
whereby cells regress from a specialized function to a simpler state reminiscent of stem cells»[6]

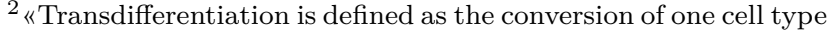
to another. It belongs to a wider class of cell type transformations called metaplasias which also includes cases in which SCs of one tissue type switch to a completely different $\mathrm{SC}$ »[27]

${ }^{3}$ «Regenerative medicine aims to replace damaged cells in the human body through a new source of healthy transplanted cells or by endogenous repair»[13]. Indeed, by artificially reprogramming adult cells (using Yamanaka Factors [28]), a new category of cells that expresses embryonic SCs characteristics is obtained: also known as induced Pluripotent Stem Cells (iPSCs).
} 
introduced in this class of systems, we begin in Section II with a general framework which serves as a basis for plasticity functions. Next, in Section III, we select a typical form of dedifferentiation mechanisms, where an unhealthy (differentiated) subpopulation of progeny cells regresses to a $\mathrm{SC}$ (cancerous) state. We highlight one of the expected cellular-plasticity effects, which is the possible survival of unhealthy cells as a result of progeny dedifferentiation. In fact, a rich behavior of the system is observed when the dedifferentiated cells are directly active in the proliferating phase, which is the common behavior of unhealthy cells when dedifferentiation is associated with cancer [30] (e.g. due to some genetic/epigenetic mutations that increase the selfrenewal activity of abnormal cells [19], [30]). A stability result is given in Section III-B, followed by a numerical example, that we discuss in Section IV.

\section{Summary and key points}

(1) In our study, we provide a general model for cell population dynamics that may describe many types of cancers. We particularly emphasize the case of hematopoietic SCs and early blood progeny (see some similar models in [20], [4], [1], [23], [12]). In fact, hematopoiesis provides a common paradigm for understanding all the mammalian SCs, in particular when it comes to study cell differentiation.

(2) We are interested in modeling a dedifferentiation scenario where a portion (i.e. a mutated subpopulation) of a given generation of differentiated progenitor cells (that belongs for instance to a specific blood lineage hierarchy as in Fig. 2, blue hierarchy), regresses into an hematopoietic stem cell proliferating state.

3 In the general case, anti-cancer therapy aims to eradicate all the malignant cancer cells. Therefore, we focus on the stability properties of the origin of our model, since it reflects extinction of malignant cells. (4) Our approach could incite modelers to systematically take into account cellular plasticity features when modeling cell population dynamics. Moreover, our result supports the idea that unhealthy cells protect themselves during anti-cancer therapy through dedifferentiation. We provide a stability condition which could help medical experts restrain -or inhibitdedifferentiation, in order to ensure cell eradication. This procedure seems in line with some medical experiments, where anti-dedifferentiation mechanisms are already used (see Section IV).

\section{Modeling CELl POPUlations AND CELLUlaR PLASTICITY: A GENERAL FRAMEWORK}

We discuss in this section a refined model (illustrated in Fig. 1-2) that highlights, qualitatively, the impact of dedifferentiation on the behavior of cancer cells. Firstly, we focus on the general form of the model in Fig. 1, in which we have $n$ distinguishable maturity levels ( $i \in$ $I_{n}=\{1, \ldots, n\}$, such that $i=1$ is the SC compartment). Models with quiescent and proliferating phases date back to some pioneer works such as Burns \& Tannock [5] and Mackey [20], which have been improved by Adimy et al.
[1], and which we extend now -as previously mentionedby adding some cell plasticity functions.

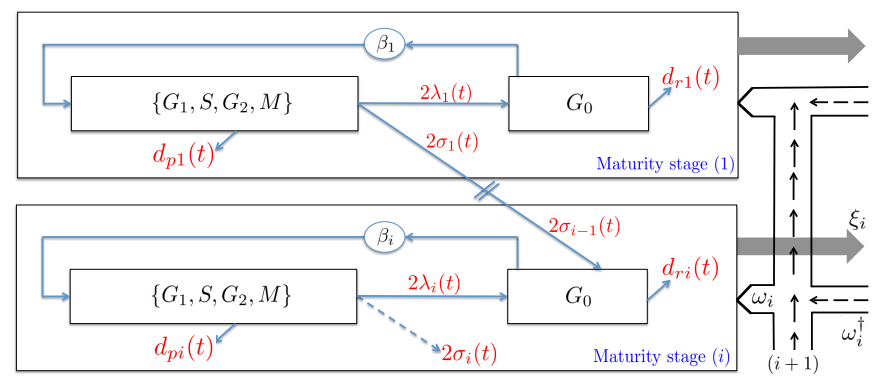

Fig. 1: A cartoon representation of the discrete maturity model of interest, involving multiple dedifferentiation and transdifferentiation functions. Cells of the $i$-th maturitygeneration, where $i \in I_{n}=\{1, \ldots, n\}, n>1$, are in a resting phase $G_{0}$, or in proliferation (cell-division cycle) $\left\{G_{1}, S, G_{2}, M\right\}$. In the general case, we can consider that all the involved biological parameters (rate of differentiation $\sigma_{i} \in(0,1)$, rate of self-renew $\lambda_{i}=1-\sigma_{i}$, apoptosis rate $d_{p i}$, death rate of resting cells $d_{r i}$ ) are time-varying. On the right, the flux due to the cell-plasticity functions, $\xi_{i}$ and $\omega_{i}$ for all $i \in I_{n}$, are represented as input or output functions. In fact, both $\xi_{i}$ and $\omega_{i}$ have a part related to dedifferentiation and another part related to transdifferentiation. The former one depends on the (modeled) state variables, which are the total densities of the resting cells $x_{i}$ as explained in Section II, while the parts quantifying trandifferentiation (denoted $\xi_{i}^{\dagger}$ and $\omega_{i}^{\dagger}$, in equations (2), (5)) are assumed to depend only on time, since they are biologically related to distant (or, external) cell tissues and lineages (i.e. which are not explicitly modeled in the specific studied cell-maturity hierarchy, see also Fig. 2).

The dynamics of resting cells, $r_{i}(t, a)$, of the $i$-th generation $\left(i \in I_{n}\right.$ ), of age $a>0$, at time $t \geq 0$, and the dynamics of proliferating cells, $p_{i}(t, a)$, of age $a \in\left[0, \tau_{i}\right]$, at time $t \geq 0$, are governed by the agestructured (McKendrick) PDEs:

$$
\left\{\begin{array}{l}
\frac{\partial p_{i}}{\partial t}+\frac{\partial p_{i}}{\partial a}=-\left[d_{p i}(t)+h_{i}(a)\right] p_{i}(t, a), \\
\frac{\partial r_{i}}{\partial t}+\frac{\partial r_{i}}{\partial a}=-\left[d_{r i}(t)+\beta_{i}\left(x_{i}(t)\right)\right] r_{i}(t, a)-\xi_{i}(t),
\end{array}\right.
$$

where, for all $i \in I_{n}, x_{i}(t)=\int_{0}^{+\infty} r_{i}(t, a) d a$, and, for all $i \in I_{n}$ and $t \geq 0, d_{r i}(t)$ is the death rate of the resting cells, while $d_{p i}(t)$ is the death rate of proliferating cells. The reintroduction function from the resting phase to the proliferative phase, $\beta_{i}$, is decreasing and $\lim _{\ell \rightarrow \infty} \beta_{i}(\ell)=$ 0 . Moreover, we consider that for all $t \geq 0$,

$$
\xi_{i}(t)=\xi_{i}(\underbrace{x_{1}(t), \ldots, x_{i-1}(t)}_{\text {out. dediff }}, x_{i}(t), \overbrace{\xi_{i}^{\dagger}(t)}^{\text {out. transdiff }}) .
$$

The system (1) is associated with some initial conditions (i.e. initial age distributions when $t=0$ ) which are $\mathcal{L}^{1}$-functions defined by:

$$
\left\{\begin{array}{l}
p_{i}(0, a)=p_{i}^{0}(a), \quad 0 \leq a \leq \tau_{i} \\
r_{i}(0, a)=r_{i}^{0}(a), \quad \text { for all } a \in[0,+\infty) .
\end{array}\right.
$$

Moreover, we assume from biological considerations that for all $t \geq 0, \lim _{a \rightarrow \infty} r_{i}(t, a)=0$. 


\section{About the functions $\xi_{i}$}

As illustrated in Fig. 2, the function $\xi_{i}$ quantifies the output (cell loss) either by dedifferentiation or by transdifferentiation. As formulated in (2), the function $\xi_{i}$ depends on $x_{i}$ and may be affected by some of, or all, the generations of cells which are less mature than the $i$-th generation, i.e. the cell densities $x_{1}, \ldots, x_{i-1}$. This is -by definition- justified by the fact that dedifferentiation of the $i$-th generation addresses a need (either healthy or cancerous) that arises in a less mature generation within the same hierarchy (i.e. at least one of the densities $x_{1}, \ldots, x_{i-1}$ ).

In addition, the loss by transdifferentiation, $\xi_{i}^{\dagger}$, aims to address a need from more distant tissues, i.e. $\xi_{i}^{\dagger}$ does not depend on the modeled cell populations $x_{i}$, but only on time (as a representation of any external event that occurs beyond the studied hierarchy).

Finally, the renewal conditions, which give the birth rate at the initial age $a=0$, are introduced through the following boundary conditions:

$$
\left\{\begin{aligned}
p_{i}(t, 0)= & \beta_{i}\left(x_{i}(t)\right) x_{i}(t)+\omega_{i}(t) \\
r_{i}(t, 0)= & 2 \sigma_{i-1}(t) \int_{0}^{\tau_{i-1}} h_{i-1}(a) p_{i-1}(t, a) d a \\
& +2\left(1-\sigma_{i}(t)\right) \int_{0}^{\tau_{i}} h_{i}(a) p_{i}(t, a) d a
\end{aligned}\right.
$$

where $\sigma_{i}(t)$ represents the time-varying rate of differentiation and, consequently, $\lambda_{i}(t)=1-\sigma_{i}(t)$ is the rate of self-renewal of the $i$-th cell generation. In addition, we consider with an abuse of notation that for all $t \geq 0$,

$$
\omega_{i}(t)=\omega_{i}(x_{i}(t), \underbrace{x_{i+1}(t), \ldots, x_{n}(t)}_{\text {in. dediff }}, \overbrace{\omega_{i}^{\dagger}(t)}^{\text {in. transdiff }}) .
$$

\section{About the functions $\omega_{i}$}

The function $\omega_{i}$ appears in the boundary conditions (4), since it represents the new cell birth resulting from the dedifferentiation of more mature cell generations, together with the transdifferentiation of other cell lineages. The functions $\omega_{i}$ are implicitly related to the functions $\xi_{i}$ in (2), since cells that join the $i$-th generation by dediffentiation (the input $\omega_{i}$ ) are, for instance, the sum of the dedifferentiated cells from more mature generations (the outputs $\xi_{i+1}, \xi_{i+2}, \ldots$ ). Therefore, for all $i \in I_{n}$, the function $\omega_{i}$ depends on $x_{i}$ and may depend on (all or some of) the more mature generations in the same hierarchy, $x_{i+1}, \ldots, x_{n}$. Moreover, the time-varying term $\omega_{i}^{\dagger}$ in (5) quantifies the incoming by trandifferentiation from distant cell lineages or types, outside the hierarchy of interest.

In summary, we say that generally in McKendrick type models, the removal terms (e.g. death rates) appear in the PDE system (model (1), in our case), while new births appear in the boundary conditions (given by (4), in our case). The PDE system (1)-(4)-(3) provides a general framework to describe the cell dynamics within a given hierarchy formed by $n$ discrete-maturity stages. However, we still need to specify the nature and the operating mode of the dedifferentiation and the transdifferentiation functions $\left(\xi_{i}\right.$ and $\omega_{i}$, for all $i \in I_{n}$ ), in order to determine the behavior of the overall system. For that purpose, we need to focus on a typical explicit dedifferentiation mechanism. More precisely, we start in this study with an unhealthy case in which a portion of the $j$-th cell generation $\left(j \in I_{n}, j>1\right)$ dedifferentiates and joins the SC compartment $(i=1)$, as presented in the sequel.

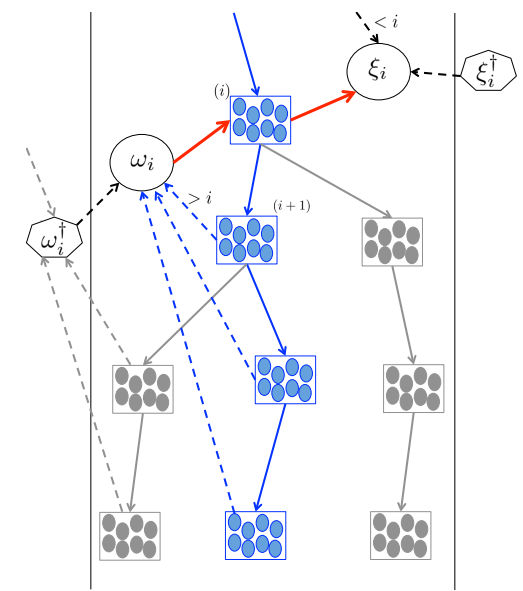

Fig. 2: The blue lineage hierarchy is the one on which we are focusing, and which is modeled by our system. More precisely, each $i$-th stage in the blue hierarchy corresponds to the $i$-th generation in the model illustrated in Fig. 1. In contrast, the gray generations/lineages are those which cannot be explicitly modeled in our mathematical framework. Next, for the $i$-th cell generation, the function $w_{i}$ quantifies the input by dedifferentiation from the upcoming more mature generations that belong to the studied blue lineage, together with the input by transdifferentiation $\left(\omega_{i}^{\dagger}\right)$, which comes from some nonmodeled distant-cell types. In a similar spirit, $\xi_{i}$ quantifies the loss in cell density of the $i$-th generation, for instance in response to some requirements from less mature generations that belong to the blue hierarchy (i.e. this is the loss due to dedifferentiation), or from other types of cells which are in gray, i.e. this is the loss/output by transdifferentiation $\left(\xi_{i}^{\dagger}\right)$.

\section{A SPECIFIC DEDIFFERENTIATION PROCESS}

We focus now on a typical case, in which a proportion $\kappa \in[0,1]$ of the total density of resting cells of the $j$ th generation $x_{j}$ becomes diseased, e.g. as a result of a series of abnormal mutations (see, e.g., [31] for mutations inducing leukemia). Then, we consider that only the subpopulation of mutated cells can undergo dedifferentiation, and we denote by $D$ the characteristic pattern that describes the dedifferentiation mechanism from the $j$-th generation into the SC-proliferating compartment.

\section{A. A model involving one dedifferentiation feedback}

Let $j \in[2, n]$ be the generation of cells that includes a malignant (mutated) subpopulation, that we assume capable of generating cancer stem cells (CSCs) by dedifferentiation (see Fig. 3 in [15]). For the sake of simplicity, we consider that the transdifferentiation mechanisms are negligible compared to dedifferentiation within the same hierarchy (i.e. we put $\xi_{i}^{\dagger}(t)=\omega_{i}^{\dagger}(t)=0$, for all $i \in I_{n}$ and $t \geq 0)$. We notice that -with an abuse of notationthe functions $\xi_{i}$ and $\omega_{i}$ can be rewritten in this case as: 


$$
\left\{\begin{array}{l}
\xi_{i}\left(x_{1}, \ldots, x_{i}\right)=0, \text { for all } i \in I_{n}, i \neq j, \\
\xi_{j}\left(x_{1}, \ldots, x_{j}\right)=\xi_{j}\left(x_{j}\right)=\kappa D\left(\kappa x_{j}\right) x_{j}, \\
\omega_{1}\left(x_{1}, \ldots, x_{n}\right)=\omega_{1}\left(x_{j}\right)=\kappa D\left(\kappa x_{j}\right) x_{j}, \\
\omega_{i}\left(x_{i}, \ldots, x_{n}\right)=0, \text { for all } i \in\{2, \ldots, n\}
\end{array}\right.
$$

where we can select the function $D$ as follows,

$$
D(\ell)=\tanh \left(\ell^{p}\right) \text {, where, } p>0, \text { for all } \ell \geq 0 .
$$

Let us say few words about the cell-plasticity functions $\xi_{i}$ and $\omega_{i}$ in (6). Firstly, we are limiting ourselves to the case of constant $\kappa$, where $\kappa \in[0,1]$. The function $D$ depends on the total density of mutated cells, which is given by $\kappa x_{j}$, since this subpopulation is the unique one that may undergo dedifferentiation. The function $D$ is selected such that $\lim _{\ell \rightarrow+\infty} D(\ell)=1$, meaning that the aggressiveness of mutated cells increases (i.e. mutated cells are more likely to dedifferentiate into CSCs) with respect to the total density of mutated cells $\kappa x_{j}$. In fact, the features of the tangent hyperbolic function $D$, as defined in (7), lead to the following qualitative behavior: (1) $\xi_{j} \equiv 0$ and $\omega_{1} \equiv 0$, if $x_{j}=0$ or $\kappa=0$, i.e. dedifferentiation does not exist without abnormal mutations.

(2) when the $j$-th cell generation forms a relatively small population over all the $n$ cell generations (i.e. a low cell density $x_{j}$ ), or when the portion of unhealthy cells is minimal within the genetic diversity landscape (i.e. a low mutated portion of cells, quantified by $\kappa$ ), then the gain of the dedifferentiation process is minimal. Indeed, the subpopulation of mutated cells is not expected to entirely dedifferentiate and form aggressive CSCs, in this case, because it is too small to impose itself upon all the other cells. We actually expect that immunological mechanisms are sufficient in this case to control the small population of mutated cells [26]. Let us notice that, in our model, the gain of the dedifferentiation process is represented by the quantity $\kappa D\left(\kappa x_{j}\right)$, since $\xi_{j}\left(x_{j}\right)=\kappa D\left(\kappa x_{j}\right) x_{j}$. So, for low $x_{j}$ and low $\kappa$, the amplitude of $\kappa D\left(\kappa x_{j}\right)$ is relatively small, then it increases in order to approach its highest value, when the total size of the population $x_{j}$ is too large, i.e. the maximum possible gain of dedifferentiation is $\kappa$ (since $\left.\lim _{x_{j} \rightarrow \infty} \kappa D\left(\kappa x_{j}\right)=\kappa\right)$.

Remark 1: The selected dedifferentiation functions (6) depend only on the mutated subpopulation of cells belonging to the $j$-th maturity generation. The latter choice can be argued as an unhealthy dedifferentiation process triggered by some abnormal mutations that occur in the $j$-th maturity stage. However, different choices may be considered within the general framework introduced in Section II. For instance, a healthy dedifferentiation process can be formulated by considering that $\xi_{i}$ and $\omega_{i}$ depend on the total density of resting SCs, $x_{1}$. In such a configuration, SCs have the ability to trigger by themselves the dedifferentiation of more mature cells, when needed (e.g. after hemorrhage or injury, when the body is in a hurry to regenerate itself). However, this healthy situation is beyond the scope of the current work.
Finally, we rewrite the model (1) describing the dynamics of resting and proliferating cells, in the form:

$$
\left\{\begin{array}{l}
\frac{\partial p_{i}}{\partial t}+\frac{\partial p_{i}}{\partial a}+\left[d_{p i}(t)+h_{i}(a)\right] p_{i}(t, a)=0 \\
\frac{\partial r_{i}}{\partial t}+\frac{\partial r_{i}}{\partial a}+\left[d_{r i}(t)+\beta_{i}\left(x_{i}(t)\right)\right] r_{i}(t, a)=0 \\
\text { for all } i \geq 1 \text { where } i \neq j, \text { and } \\
\frac{\partial p_{j}}{\partial t}+\frac{\partial p_{j}}{\partial a}+\left[d_{p j}(t)+h_{j}(a)\right] p_{j}(t, a)=0 \\
\frac{\partial r_{j}}{\partial t}+\frac{\partial r_{j}}{\partial a}+\left[d_{r j}(t)+\beta_{j}\left(x_{j}(t)\right)\right] r_{j}(t, a) \\
+\kappa D\left(\kappa x_{j}(t)\right) x_{j}(t)=0
\end{array}\right.
$$

We emphasize the case of (4) described throughout the current section, in which the renewal conditions are introduced through the following boundary conditions:

$$
\left\{\begin{aligned}
p_{1}(t, 0)= & \beta_{1}\left(x_{1}(t)\right) x_{1}(t)+\kappa D_{j}\left(\kappa x_{j}(t)\right) x_{j}(t), \\
p_{i}(t, 0)= & \beta_{i}\left(x_{i}(t)\right) x_{i}(t), \text { for all } i \in\{2, \ldots, n\} \\
r_{1}(t, 0)= & 2\left(1-\sigma_{1}(t)\right) \int_{0}^{\tau_{1}} h_{1}(a) p_{1}(t, a) d a \\
\text { and for all } i>1: & \\
r_{i}(t, 0)= & 2\left(1-\sigma_{i}(t)\right) \int_{0}^{\tau_{i}} h_{i}(a) p_{i}(t, a) d a \\
& +2 \sigma_{i-1}(t) \int_{0}^{\tau_{i-1}} h_{i-1}(a) p_{i-1}(t, a) d a
\end{aligned}\right.
$$

Finally, we associate to (8)-(9) some suitable initial conditions similar to (3). Next, using the method of characteristics (see similar applications in [4] and [1]), we can reduce the model (8)-(9)-(3) into a nonlinear system with finite distributed delays and time-varying parameters. For the sake of clarity, we illustrate these statements in the case involving two cell-maturity generations, in which all the biological parameters are constant and a dedifferentiation mechanism is established from the 2 nd generation into the SC one, as shown in Fig. 3.

For instance, we consider that a progeny subpopulation $\kappa x_{2}$, where $\kappa \in[0,1]$, has some abnormal mutations (e.g. DMNT3A increasing the self-renewal activity), that may trigger dedifferentiation into CSCs [8], as it appears to be the case in leukemia [30], [19]. The time-delay version of the model of interest is given by:

$$
\left\{\begin{array}{l}
\dot{x}_{1}(t)=-\left[d_{r 1}+\beta_{1}\left(x_{1}(t)\right)\right] x_{1}(t) \\
\quad+2\left(1-\sigma_{1}\right) \int_{0}^{\tau_{1}} g_{1}(a) \beta_{1}\left(x_{1}(t-a)\right) x_{1}(t-a) d a \\
\quad+2\left(1-\sigma_{1}\right) \int_{0}^{\tau_{1}} g_{1}(a) \kappa x_{2}(t-a) D\left(\kappa x_{2}(t-a)\right) d a \\
\dot{x}_{2}(t)=-\left[d_{r 2}-\beta_{2}\left(x_{2}(t)\right)-\kappa D\left(\kappa x_{2}(t)\right)\right] x_{2}(t) \\
\quad+2\left(1-\sigma_{2}\right) \int_{0}^{\tau_{2}} g_{2}(a) \beta_{2}\left(x_{2}(t-a)\right) x_{2}(t-a) d a \\
\quad+2 \sigma_{1} \int_{0}^{\tau_{1}} g_{1}(a) \beta_{1}\left(x_{1}(t-a)\right) x_{1}(t-a) d a \\
\quad+2 \sigma_{1} \int_{0}^{\tau_{1}} g_{1}(a) \kappa x_{2}(t-a) D\left(\kappa x_{2}(t-a)\right) d a
\end{array}\right.
$$

where $f_{i}(\ell)=h_{i}(\ell) e^{-\int_{0}^{\ell} h_{i}(m) d m}$ and $g_{i}(\ell)=f_{i}(\ell) e^{-d_{p i} \ell}$, (see [1] for a similar application, but also [4]). In addition, we can notice that the system (10) is positive.

\section{B. Sufficient global asymptotic stability conditions}

Now, we want to determine stability conditions of the origin of the model (10). Convergence of the model trajectories to zero is equivalent to total cell eradication, e.g. thanks to anti-cancer infusions therapy. Firstly, let 
us define for later use the constants: $s_{i}=\delta_{i}-\alpha_{i} \beta_{i}(0)$, $\alpha_{i}=2 \lambda_{i} C_{i}-1$, for all $i \in I_{n}$, and,

$$
\mathfrak{a}=s_{2}-\kappa \bar{D}\left[\frac{2 \sigma_{1} \lambda_{1} C_{1}^{2} \beta_{1}(0)}{s_{1}}+2 \sigma_{1} C_{1}-1\right],
$$

where, $\bar{D}=\sup _{\ell \geq 0} D(\ell)$. Notice that, $\bar{D}=1$, when, $D(\ell)=\tanh \left(\ell^{p}\right), p>0$. It is worth mentioning that for a model without dedifferentiation (i.e. when $\kappa=0$ ), the conditions $s_{i}>0$, for all $i \in I_{n}$ are necessary and sufficient conditions for global exponential stability of the origin of the corresponding system (see [1] and [11]). We state in this paper -without details of the proof- the following stability result:

Theorem 1: Let us assume that $s_{i}>0$ for all $i \in I_{n}$. If, in addition, the conditions,

$\left\{\begin{array}{l}1-2 \sigma_{1} C_{1}-\frac{2 \sigma_{1} \lambda_{1} C_{1}^{2} \beta_{1}(0)}{s_{1}}<0, \text { and, } \mathfrak{a}>0, \\ \text { or, } 1-2 \sigma_{1} C_{1}-\frac{2 \sigma_{1} \lambda_{1} C_{1}^{2} \beta_{1}(0)}{s_{1}} \geq 0,\end{array}\right.$

are satisfied, then the origin of the model (10) is globally asymptotically stable.

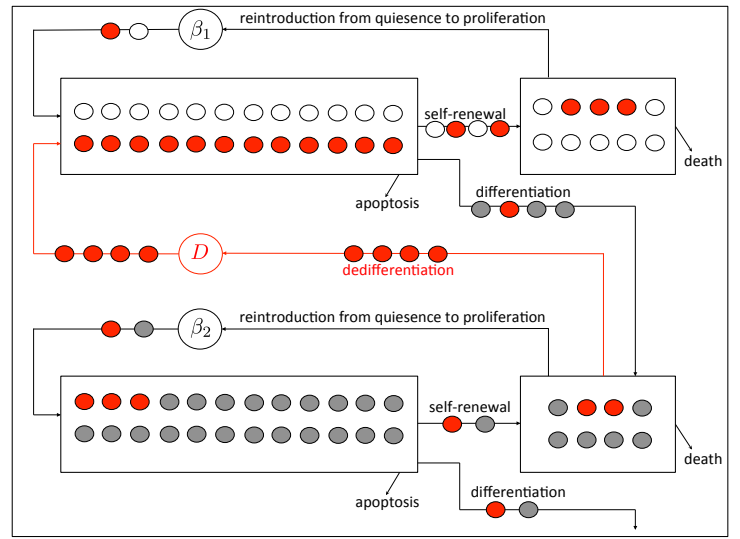

Fig. 3: Red cells are malignant (mutated) cells. White cells are ordinary stem cells (SCs), while the gray cell compartment is the one of (progeny) differentiated cells, that includes the subpopulation (in red) $\kappa x_{2}$. A mechanism of dedifferentiation of malignant cells (in red, from $i=2$ to $i=1$ ) is established through the function $D$. In hematopoiesis, a translocation effect after some epigenetic mutations in the progeny compartment (here $i=2$ ) may lead to a dedifferentiation of progeny ([8]) and the rise of cancer stem cells (CSCs), that trigger a quick progression of leukemia [30]. For instance, a DNMT3A mutation may provide abnormal cells with a selfrenewal activity as important as the one of SCs [19], [30].

Remark 2: In fact, the model (10) may admit a positive steady state even if $s_{i}>0$, which is not the case when $\kappa=0$. Therefore, we notice that then conditions $s_{i}>0$, for all $i \in I_{n}$ are no longer sufficient to ensure that the 0 -equilibrium is globally stable when dedifferentiation exists. In light of Theorem 1, we deduce that zero is stable if we ensure that an upper-bound on $\kappa$ is satisfied, i.e. if dedifferentiation does not cross the threshold defined by the condition $\mathfrak{a}>0$ in (12). Medical practice supports this observation ([16], [25]), as discussed in the sequel.

\section{A NUMERICAL ILLUSTRATION AND SOME CONCLUDING COMMENTS}

Medical practice shows that sometimes if dedifferentiation is neglected, cancer cells may survive to therapy. We consider the concrete situation studied in [16], [25].

\section{A glimpse into the medical experience}

In [16], some experiences have been conducted on human non-small cell lung cancer. Their results suggested that non-stem cancer cells which were targeted through radiotherapy, have protected themselves by dedifferentiation processes (CSCs are particularly resistant to radiotherapy, see for example [3], [9]). In [25], an ODE-model was proposed to fit the data of [16]. In a second time, the experience was renewed by adding some survivin inhibitors (known as YM155), that undermined dedifferentiation of cancer cells. The therapy efficacy was substantially improved when YM155 was used along with radiotherapy [25], [16].

We check the qualitative behavior of our system through some situations that are reflective of those encountered in [16], [25]. For that purpose, we consider for instance the cell division probability densities of the forms $f_{i}(a)=\frac{m_{i}}{e^{m_{i} \tau_{i}}-1} e^{m_{i} a}$, with $m_{i}>0, i \in I_{n}$, along with the following functions and parameters:

\begin{tabular}{|c|c|c|c|c|c|c|}
\hline & $\beta_{i}\left(x_{i}\right)$ & $f_{i}(a)$ & $d_{r i}$ & $\lambda_{i}$ & $\tau_{i}$ & $d_{p i}$ \\
\hline$i=1$ & $\frac{1.11}{1+x_{1}^{2}}$ & $\frac{10 e^{10 a}}{e^{10 \tau}-1}$ & 0.112 & 0.7 & 1.19 & 0.27 \\
\hline$i=2$ & $\frac{1.4}{1+x_{2}^{4}}$ & $\frac{10 e^{10 a}}{e^{10 \tau}-1}$ & 0.37 & 0.9 & 1.32 & 0.33 \\
\hline
\end{tabular}

After simple calculations, we get: $s_{1}=0.0518$ and $s_{2}=$ 0.2218 . Therefore, in the case where dedifferentiation does not exist (i.e. $\kappa=0$ ), the origin of the studied model is globally exponentially stable [11], [1], as illustrated in Fig. 4-(a). Next, let us assume that at $t=20$ days, the dedifferentiation mechanism is triggered (by setting $\kappa=0.8$ at $t=20$ days). In that case, we still have $s_{1}>0$ and $s_{2}>0$, but, however, we note that the sufficient stability conditions (12), in Theorem 1 , are not satisfied:

$$
\begin{aligned}
& 1-2 \sigma_{1} C_{1}-\frac{2 \sigma_{1} \lambda_{1} C_{1}^{2} \beta_{1}(0)}{s_{1}}=-4.45, \text { and, } \\
& \mathfrak{a}=s_{2}-\kappa \bar{D}\left[\frac{2 \sigma_{1} \lambda_{1} C_{1}^{2} \beta_{1}(0)}{s_{1}}+2 \sigma_{1} C_{1}-1\right]=-3.33 .
\end{aligned}
$$

In simulation, we observe that for some initial conditions, the origin of the system (10) is not asymptotically stable, as illustrated in Fig. 4-(b). More importantly, we notice that the trajectories converge in this case to a strictly positive steady state, given by $x_{1}^{*}=1.47$ and $x_{2}^{*}=0.66$, that exists even if $s_{i}>0$ (contrary to the case $\kappa=0$, where zero is the unique steady state when $\left.s_{i}>0\right)$. In addition, we can determine the gain of the dedifferentiation function, which converges to $\kappa D\left(\kappa x_{2}^{*}\right)=$ 0.12 , when the trajectories of the system (10) approach the positive steady state. Thus, in this example, the unhealthy cells prevent themselves from total extinction thanks to their dedifferentiation ability (Fig. 4 (a)-(b)). Finally, we notice in Fig. 4-(c) that for some sufficiently 


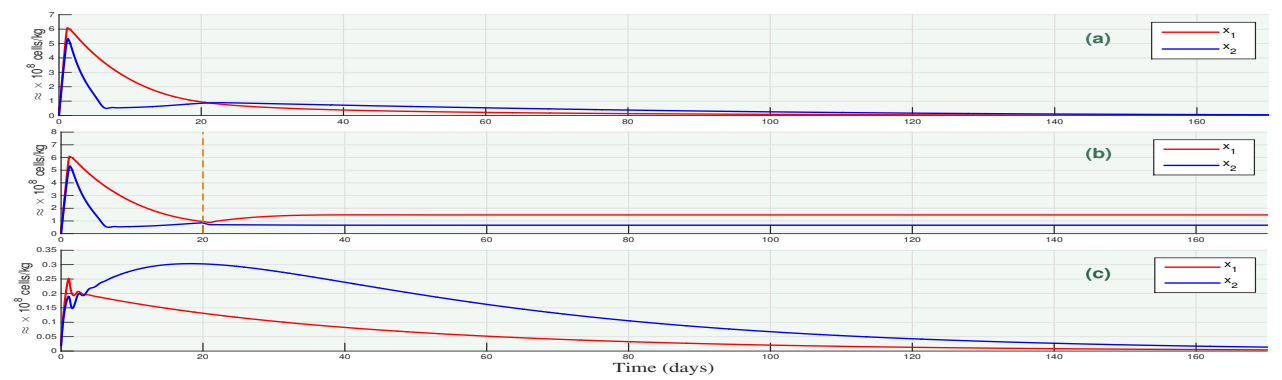

Fig. 4: In (a), $\kappa=0, s_{i}>0$, the stability conditions are satisfied and we observe that the trajectories converge to zero. In (b) and $(\mathbf{c})$, dedifferentiation processes are triggered at $t=20$. In (b), the trajectories do not converge to zero (they converge to a small positive steady state), meaning that dedifferentiation prevents cells from total extinction. This observation supports the hypothesis claiming that cancer cells may protect themselves through dedifferentiation during anti-cancer therapy [13]. We suggest that therapeutics should take into account cell plasticity features, by including drugs that inhibit the dedifferentiation mechanisms, in order to satisfy the conditions given in Theorem 1 . In other words, we want to design a therapy that avoids the case described in (b). Otherwise, malignant cells may regenerate themselves after therapy. In (c), dedifferentiation is also triggered at $t=20$, but this time, we consider that the initial mutated subpopulation $\kappa x_{j}$ is relatively too small. In this case, dedifferentiation is ineffective in ensuring unhealthy cell survival (mutated cells disappear over time without creating an aggressive cancer). Sometimes it is suggested that immmunological mechanisms (immunoediting [26]) may prevent mutated cells from the outgrowth that characterizes CSCs (i.e. the immune system acts to restrain the gain of dedifferenciation mechanisms).

small initial conditions and $\kappa=0.8$, the dedifferentiation process is not sufficient to avoid the total cell eradication.

\section{REFERENCES}

[1] Adimy, M., Crauste, F., ElAbdllaoui, A., 2008. Discretematurity structured model of cell differentiation with applications to acute myelogenous leukemia. Journal of Biological Systems, 16(03), pp.395-424.

[2] Apostu, R. and Mackey, M.C., 2008. Understanding cyclical thrombocytopenia: A mathematical modeling approach. Jour. Theor. bio., 251(2), pp.297-316.

[3] Bao, S., et al., 2006. Glioma stem cells promote radioresistance by preferential activation of the DNA damage response. Nature, 444(7120), p.756.

[4] Bélair, J., Mackey, M.C. and Mahaffy, J.M., 1995. Agestructured and two-delay models for erythropoiesis. Mathematical biosciences, 128(1-2), pp.317-346.

[5] Burns, F., and Tannock I., 1970. On the existence of a $G_{0}$ phase in the cell cycle. Cell Tissue Kinet, Vol.3, pp.321-334.

[6] Cai, S.A., Fu, X. and Sheng, Z., 2007. Dedifferentiation: a new approach in stem cell research. AIBS Bulletin, 57(8), p.655.

[7] Cooper, G. M., and Hausman, R. E., 2015. The Cell: A Molecular Approach. Published by Sinauer Associates, Inc., Seventh Edition, p.832.

[8] Cozzio, A., Passegué, E., et al., 2003. Similar MLL-associated leukemias arising from self-renewing stem cells and short-lived myeloid progenitors. Genes \& development, 17(24), p.3029.

[9] Dahan, P., et al., 2014. Ionizing radiations sustain glioblastoma cell dedifferentiation to a stem-like phenotype through survivin: possible involvement in radioresistance. Cell death $\&$ disease, 5(11), p.e1543.

[10] Dale, D.C., Mackey, M.C., 2015. Understanding, treating and avoiding hematological disease: Better medicine through mathematics? Bull. Math. Bio. 77(5), p.739.

[11] Djema, W., Mazenc, F. and Bonnet, C., 2017. Stability analysis and robustness results for a nonlinear system with distributed delays describing hematopoiesis. Systems \& Control Letters, 102, pp.93-101.

[12] Djema, W., Bonnet, C., Clairambault, J., Mazenc, F., Hirsch, P., Delhommeau, F., 2017. Analysis of a model of dormancy in cancer as a state of coexistence between tumor and healthy stem cells. American Control Conference (ACC), p.5135.

[13] Doherty, et al., 2016. Cancer stem cell plasticity drives therapeutic resistance. Cancers, 8(1), p.8.

[14] Enver, T., Pera, M., Peterson, C. and Andrews, P.W., 2009. Stem cell states, fates, and the rules of attraction. Cell stem cell, 4(5), pp.387-397.
[15] Friedman-Morvinski, D. and Verma, I.M., 2014. Dedifferentiation and reprogramming: origins of cancer stem cells. EMBO reports, p.244.

[16] Iwasa, T., et al., 2008. Radiosensitizing effect of YM155, a novel small-molecule survivin suppressant, in non-small cell lung cancer cell lines. Clin. Canc. Res., 14(20), pp.6496-6504.

[17] Jilkine, A. and Gutenkunst, R.N., 2014. Effect of dedifferentiation on time to mutation acquisition in stem cell-driven cancers. PLoS computational biology, 10(3), p.e1003481.

[18] Leder, K., Holland, E.C. and Michor, F., 2010. The therapeutic implications of plasticity of the cancer stem cell phenotype. PloS one, 5(12), p.e14366.

[19] Ley, T.J., et al., 2010. DNMT3A mutations in acute myeloid leukemia. N. Engl. J. Med., 363(25), pp.2424-2433.

[20] Mackey, M.C., 1978. Unified hypothesis for the origin of aplastic anemia and periodic hematopoiesis. Blood, 51(5), p.941.

[21] Mackey, M.C. and Maini, P.K., 2015. What has mathematics done for biology?. Bull. Math. Bio., 77(5), p.735.

[22] Murray, J.D., 2002. Mathematical Biology: I: An Introduction. Third Edition, Interdisciplinary Applied Mathematics, Mathematical Biology, New York: Springer, pp.551.

[23] Özbay, H., Bonnet, C., Benjelloun, H. and Clairambault, J., 2012. Stability analysis of cell dynamics in leukemia. Mathematical Modelling of Natural Phenomena, 7(1), pp.203-234.

[24] Reya, T., et al., 2001. Stem cells, cancer, and cancer stem cells. nature, 414(6859), p.105.

[25] Rhodes, A. and Hillen, T., 2016. Mathematical Modeling of the Role of Survivin. Bull. Math. Bio. 78(6), p.1162.

[26] Schreiber, R.D., Old, L.J., Smyth, M.J., 2011. Cancer immunoediting: integrating immunity's roles in cancer suppression and promotion, Science, 331(6024), pp. 1565-1570.

[27] Shen, C.N., Burke, Z.D., Tosh, D., 2004. Transdifferentiation, metaplasia and tissue regeneration. Organogenesis, 1(2), p.36.

[28] Takahashi, K., Tanabe, et al., 2007. Induction of pluripotent stem cells from adult human fibroblasts by defined factors. cell, 131(5), pp.861-872.

[29] Tata, P.R. and Rajagopal, J., 2016. Cellular plasticity: 1712 to the present day. Current Opinion in Cell Biology, 43, pp.46-54.

[30] Tung, P.Y., Knoepfler, P.S., 2015. Epigenetic mechanisms of tumorigenicity manifesting in SCs. Oncogene, 34(18), p.2288.

[31] Xie, M., et al., 2014. Age-related mutations associated with clonal hematopoietic expansion and malignancies. Nature medicine, 20(12), pp.1472-1478.

[32] Yamada, Y., Haga, H. and Yamada, Y., 2014. Concise review: dedifferentiation meets cancer development: proof of concept for epigenetic cancer. Stem cells translational medicine, 3(10), pp.1182-1187. 